\title{
Cardiovascular and connective tissue disorder features in FLNA-related PVNH patients: progress towards a refined delineation of this syndrome
}

\author{
Clarisse Billon ${ }^{1,2^{*}}\left(\mathbb{0}\right.$, Salma Adham ${ }^{1,3 \dagger}$, Natalia Hernandez Poblete ${ }^{4,5 \dagger}$, Anne Legrand ${ }^{1,2}$, Michael Frank ${ }^{1,2}$, \\ Laurent Chiche ${ }^{6,7}$, Stephane Zuily ${ }^{8,9}$, Karelle Benistan ${ }^{10,11}$, Laurent Savale ${ }^{12,13,14}$, Khaoula Zaafrane-K \\ hachnaoui ${ }^{15}$, Anne-Claire Brehin ${ }^{16}$, Laurence Bal ${ }^{17}$, Tiffany Busa $^{18}$, Mélanie Fradin ${ }^{19}$, Chloé Quelin ${ }^{19}$, \\ Bertrand Chesneau 20,21, Denis Wahl ${ }^{8,9}$, Patricia Fergelot ${ }^{4,5}$, Cyril Goizet ${ }^{4,6}$, Tristan Mirault ${ }^{1,2}$, Xavier Jeunemaitre ${ }^{1,2}$, \\ Juliette Albuisson 1,2,22 and Bordeaux-cohort collaborators
}

\begin{abstract}
Background: FLNA Loss-of-Function (LoF) causes periventricular nodular heterotopia type 1 (PVNH1), an acknowledged cause of seizures of various types. Neurological symptoms are inconstant, and cardiovascular (CV) defects or connective tissue disorders (CTD) have regularly been associated. We aimed at refining the description of CV and CTD features in patients with FLNA LOF and depicting the multisystemic nature of this condition.
\end{abstract}

Methods: We retrospectively evaluated FLNA variants and clinical presentations in FLNA LoF patient with at least one CV or CTD feature, from three cohorts: ten patients from the French Reference Center for Rare Vascular Diseases, 23 patients from the national reference diagnostic lab for filaminopathies-A, and 59 patients from literature review.

Results: Half of patients did not present neurological symptoms. Most patients presented a syndromic association combining CV and CTD features. CV anomalies, mostly aortic aneurysm and/or dilation were present in 75\% of patients. CTD features were present in $75 \%$. Variants analysis demonstrated an enrichment of coding variants in the $\mathrm{CH} 1$ domain of FLNA protein.

Conclusion: In FLNA LoF patients, the absence of seizures should not be overlooked. When considering a diagnosis of PVNH1, the assessment for CV and CTD anomalies is of major interest as they represent interlinked features. We recommend systematic study of FLNA within CTD genes panels, regardless of the presence of neurological symptoms.

Keywords: FLNA, Connective tissue disorder, Aortic aneurysm and dissection, Cardiovascular anomalies, EhlersDanlos

\footnotetext{
*Correspondence: clarisse.billon@aphp.fr

†Salma Adham and Natalia Hernandez Poblete have contributed equally to this work

${ }^{1}$ Département de génétique, Centre national de référence pour les maladies vasculaires rares, centre de référence européen VASCERN MSA, Hôpital Européen Georges Pompidou, AP-HP, 20 rue Leblanc, 75015 Paris, France

Full list of author information is available at the end of the article List of authors and their affiliations appears in the Acknowledgements
}

\section{Background}

Pathogenic variants in the X-linked FLNA gene encoding Filamin-A, a widely expressed cytoskeletal protein, lead to highly variable clinical presentations including periventricular nodular heterotopia type 1 (PVNH1, OMIM \#300049). PVNH1 is an acknowledged cause of seizures $[1,2]$ and can be associated with cardiovascular $(\mathrm{CV})$ original author(s) and the source, provide a link to the Creative Commons licence, and indicate if changes were made. The images or other third party material in this article are included in the article's Creative Commons licence, unless indicated otherwise in a credit line to the material. If material is not included in the article's Creative Commons licence and your intended use is not permitted by statutory regulation or exceeds the permitted use, you will need to obtain permission directly from the copyright holder. To view a copy of this licence, visit http://creativecommons.org/licenses/by/4.0/. The Creative Commons Public Domain Dedication waiver (http://creativeco mmons.org/publicdomain/zero/1.0/) applies to the data made available in this article, unless otherwise stated in a credit line to the data. 
anomalies including patent ductus arteriosus (PDA) and thoracic aortic dilation involving the Valsalva sinuses or tubular aorta. The disease is X-linked dominantly inherited and essentially affects women carrying heterozygous loss-of-function (LoF) pathogenic variants. A few hemizygous men with hypomorphic pathogenic variant or somatic mosaicism have also been described [3-6]. To date, published LoF pathogenic variants are found all along the protein and their location is not predictive of the severity of the phenotype. Filamin-A is composed with an actin-binding domain consisting of an $\mathrm{N}$ terminal domain (Nter) and two calponin homology domains (CH1 and $\mathrm{CH} 2)$ as well as two ROD regions (ROD1 and ROD2) composed of Immunoglobulin-like (Ig-like) repeats ( 1 to 15 and 16 to 23 respectively) [7, 8]. One intervening calpain-sensitive "hinge" sequence is located between these two ROD domains (Hinge1). C-terminal domain (Cter), where dimerization of filamin is mediated, is defined with the Ig-like repeat 24 and one "hinge" sequence located between Ig-like repeats 23 and 24 (Hinge 2) [8].

In 2005, Sheen et al. [9] suggested that pathogenic FLNA variants could lead to connective tissue disorders (CTD) through a description of two families and nine sporadic cases with periventricular nodular heterotopia (PNH) associated with joint hyperlaxity (JHL), skin hyperelasticity (SHE) and aortic aneurysm/dissection. The authors suggested calling this condition Ehlers-Danlos variant of periventricular heterotopia. Further articles reported FLNA-mutated patients, presenting with $\mathrm{PNH}$, JHL, SHE and other clinical features suggestive of CTD, supporting Sheen's hypothesis $[1,10]$. Hence, PVNH1 previously belonged to the Ehlers-Danlos syndromes (EDSs) family as Ehlers-Danlos syndrome (EDS) with periventricular heterotopia (EDS-PNH) [9-11]. This phenotype was excluded from the 2017 EDS international classification [12], due to predominant neurological presentation in the patients and insufficient data to differentiate PVNH1 from EDS-PNH.

Patients with CV anomalies and CTD phenotypes, sometimes associated with neurological symptoms, have been regularly referred to the National Reference Center for Rare Vascular Diseases, a tertiary care center, and finally diagnosed with PVNH1. These repeated observations led us to address the question of the frequency and distribution of CV and CTD features besides neurological clinical findings in patients carrying FLNA LoF variants. To answer that question we used a multiple approach strategy. First we described the characteristics of patients carrying FLNA LoF variants in whom genetic testing was performed because of CV or CTD features. Then we performed a comparison with the largest French cohort of PVNH1 patients, from the National Reference Diagnostic lab for filaminopathies A at Bordeaux University Hospital. Finally, we conducted a rapid review of the published literature on PVNH1 patients displaying CV and CTD features.

\section{Results \\ Cohort 1}

FLNA pathogenic variants were identified in 10 female Index-Cases of median age 38 years [range 14-66] (Table 1). Detailed phenotypic description is available in Table 1 and Additional file 1: Data S1.

PNH was proven in all nine patients who had cerebral MRI. Only five patients presented seizures (Table 1). All patient presented CV and CTD features with a median of 3 [min-max: 3-5] CV anomalies and 3 [min-max: 1-6] CTD features (Fig. 1A, Additional file 2: Data S2). The most frequent $\mathrm{CV}$ anomalies were aortic dilatation/ aneurysm in nine patients, followed by mitral valve anomaly in six (Table 1, Figs. 1B and 2A). One patient had severe varicosities of the feet (Index-case \#3, Fig. 2A). The two major CTD features, JHL and SHE were present in seven and four patients respectively (Fig. 1B). All patients presented a syndromic presentation with at least four clinical features belonging to CTD spectrum associated with rare vascular disorders (Fig. 1A, C). This presentation was highly variable amongst index-cases (Fig. 1A, B). Family history of these patients also demonstrated a high intrafamilial phenotypic variability.

To illustrate intrafamilial and inter-individual variability, we shortly present Index-Case\#1 and pedigree \#9 (Fig. 2B). Index-case \# 1 was a 38-year-old female referred to investigate a syndromic aortopathy. She had a thoracic ascending aorta aneurysm (TAAA, Sinus of Vasalva $\mathrm{Z}$-score $=4.13$ ) and an aortic dissection developed on an abdominal aortic aneurysm. Several CTD features were noted after physical examination (Additional file 1: Data S1 and Fig. 2A), as well as a severe panlobular emphysema of unknown origin, not related to smoking. Family history revealed that her mother died at 62 years from a bicuspid aortic valve complicated with type A aortic dissection with a $140 \mathrm{~mm}$ diameter ascending aortic aneurysm at hospital admission. Personal or family history of epilepsy was absent. This severe and syndromic presentation in two first-degree related women without risk factors led us to perform CTD genetic analysis. A motherly inherited FLNA LoF variant c.705G>A, p.(Trp235*) was identified. Cerebral MRI was then carried out and evidenced $\mathrm{PNH}$ and mega cisterna magna. Pedigree \#9 is also representative of high intrafamilial phenotypic variability (Fig. 2B). 
Table 1 Frequency of clinical neurological, cardiovascular and connective tissue disorder features in the three cohorts

\begin{tabular}{|c|c|c|c|}
\hline & $\begin{array}{l}\text { Cohort } 1(n=10) \\
\%(n)\end{array}$ & $\begin{array}{l}\text { Cohort } 2(n=23) \\
\%(n)\end{array}$ & $\begin{array}{l}\text { Literature } \\
\text { patients } \\
(n=59) \\
\%(n)\end{array}$ \\
\hline \multicolumn{4}{|l|}{ Neurological findings } \\
\hline Age & $38[14-66]$ & $16[1-60]$ & $15[0-71]$ \\
\hline Seizures & $50 \%(5 / 10)$ & $70 \%(16 / 23)$ & $46 \%(26 / 56)$ \\
\hline Mega cisterna magna & $29 \%(2 / 7)$ & $25 \%(5 / 20)$ & $31 \%(14 / 45)$ \\
\hline \multicolumn{4}{|l|}{ Cardiovascular findings } \\
\hline Aortic dilation/aneurysm & $90 \%(9 / 10)$ & $67 \%(10 / 15)$ & $51 \%(27 / 53)$ \\
\hline Pulmonary artery dilation/PAH & $38 \%(3 / 8)$ & $20 \%(2 / 10)$ & $39 \%(18 / 46)$ \\
\hline Aortic valve dysfunction & $44 \%(4 / 9)$ & $50 \%(10 / 20)$ & $20 \%(10 / 50)$ \\
\hline Mitral valve anomalies & $67 \%(6 / 9)$ & $11 \%(2 / 18)$ & $41 \%(19 / 46)$ \\
\hline Tricuspid valve anomalies (Prolapse, dysplasia) & $11 \%(1 / 9)$ & $6 \%(1 / 16)$ & $25 \%(11 / 44)$ \\
\hline Bicuspid aortic valve & $0 \%(0 / 8)$ & $29 \%(5 / 17)$ & $3 \%(1 / 39)$ \\
\hline PDA & $0 \%(0 / 8)$ & $27 \%(3 / 11)$ & $41 \%(21 / 51)$ \\
\hline VSD/ASD & $22 \%(2 / 9)$ & $100 \%(2 / 2)$ & $27 \%(13 / 48)$ \\
\hline Early-onset varicose veins & $33 \%(3 / 9)$ & $36 \%(4 / 11)$ & $100 \%(1 / 1)$ \\
\hline Arterial tortuosity & $11 \%(1 / 9)$ & $0 \%(0 / 5)$ & $60 \%(3 / 5)$ \\
\hline Arterial aneurysm/dissection & $11 \%(1 / 10)$ & $18 \%(2 / 11)$ & $60 \%(3 / 5)$ \\
\hline \multicolumn{4}{|l|}{ Connective tissue disorder features } \\
\hline Joints hyperlaxity (JHL) & $70 \%(7 / 10)$ & $53 \%(9 / 17)$ & $73 \%(29 / 40)$ \\
\hline Skin hyperelasticity (SHE) & $44 \%(4 / 9)$ & $33 \%(5 / 15)$ & $42 \%(14 / 33)$ \\
\hline Spontaneous or easily bruising & $38 \%(3 / 8)$ & $23 \%(3 / 13)$ & $24 \%(4 / 17)$ \\
\hline Cutaneous fragility (scar) & $38 \%(3 / 8)$ & $8 \%(1 / 12)$ & $63 \%(10 / 16)$ \\
\hline Wall hernia & $11 \%(1 / 9)$ & $20 \%(3 / 15)$ & $53 \%(9 / 17)$ \\
\hline Gastro-intestinal problems & $13 \%(1 / 8)$ & $17 \%(2 / 12)$ & $63 \%(12 / 19)$ \\
\hline Emphysema & $44 \%(4 / 9)$ & $7 \%(1 / 15)$ & $58 \%(19 / 33)$ \\
\hline PNO & $0 \%(0 / 10)$ & $0 \%(0 / 16)$ & $20 \%(2 / 10)$ \\
\hline Scoliosis & $60 \%(6 / 10)$ & $28 \%(5 / 18)$ & $18 \%(3 / 17)$ \\
\hline Pectus carinatum/excavatum & $0 \%(0 / 10)$ & $0 \%(0 / 16)$ & $25 \%(4 / 16)$ \\
\hline
\end{tabular}

\section{Cohort 2}

Among the 111 female Index-Cases with PVNH1, 48\% $(\mathrm{n}=53)$ had seizures. We selected the 23 patients for whom at least one CV or CTD feature was mentioned in the clinical database of the lab. For $79 \%(n=88)$ the status related to CV or CTD abnormalities was unknown.

Based on HGVS and ACMG classification [13] all the variants excepted one were classified pathogenic or likely pathogenic. Complementary functional analyses for one variant (patient \#2) was performed: FLNA cDNA sequencing on blood cells demonstrating that c.2625G $>A$ produced a $34 \mathrm{bp}$ deletion shifting the reading frame,
(p.Lys876SerfsTer11) and led us to classify this synonymous variant as pathogenic (Additional file 5: Method $\mathrm{S} 1$ ). The synonymous variant in patient \#20 was classified as VUS (c.5184C>T p.(Gly1728Gly)), RNA analysis was not performed.

The median age of these 23 patients was 16 years [minmax: 0-60] and their detailed phenotypic description is presented in Table 1 and Additional file 3: Data S3. Most patients were within infancy when clinical description was provided.

All 23 patients had $\mathrm{PNH}$ demonstrated before molecular diagnosis and $70 \%$ of patients $(n=16)$ had seizures.

(See figure on next page.)

Fig. 1 Distribution of clinical features among patients from the three cohorts. A Bar chart of cumulated CV or CD features in patients from the three cohorts. Most patients present two or more features of each field. B Radar plots summarizing cardiovascular (left panel) and Connective tissue disorder (right panel) features of the 3 cohorts. C Euler diagrams of clinical overlap between neurological, cardiovascular and EDS anomalies in each cohort 


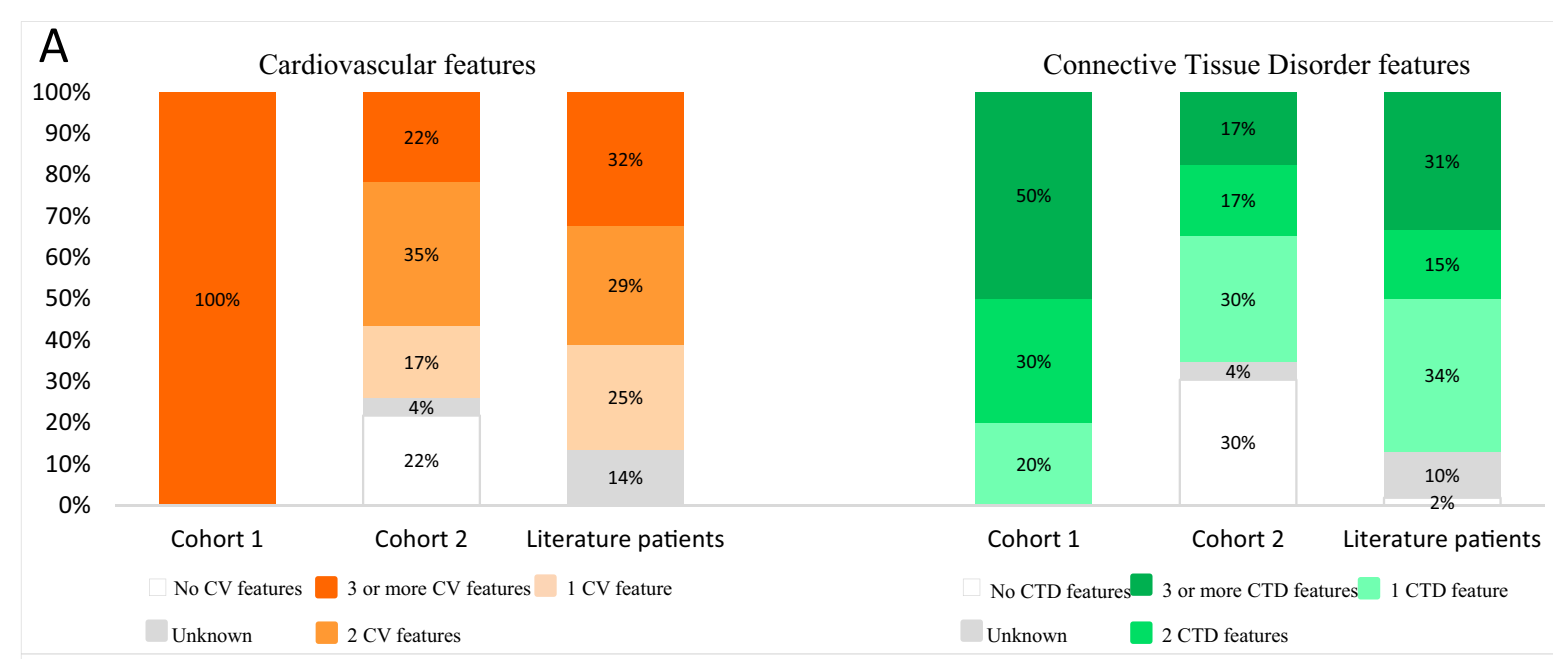

B

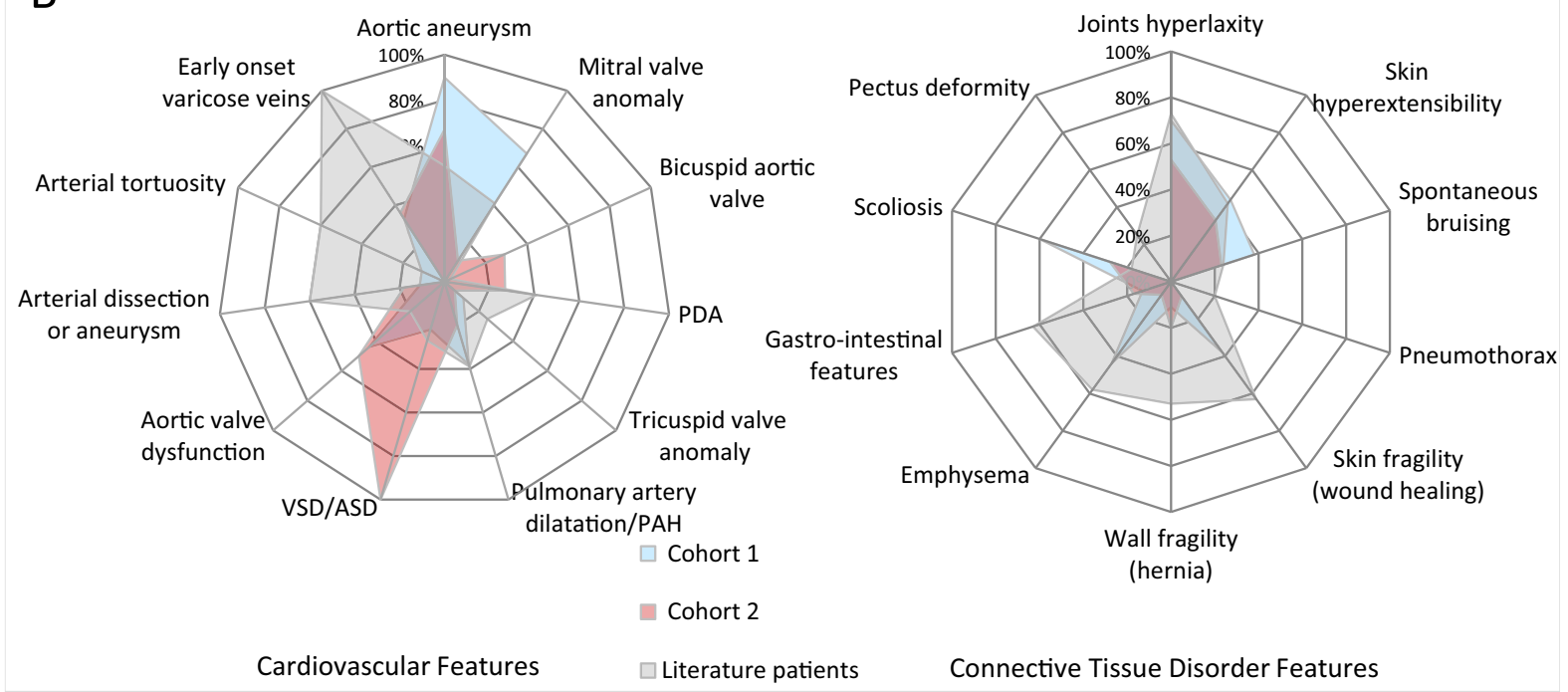

C

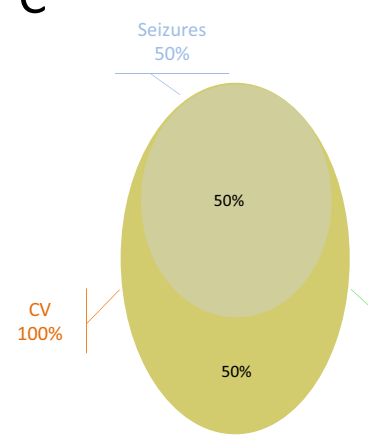

Cohort 1

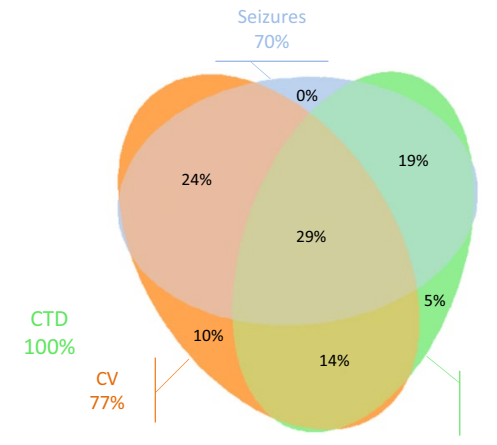

Cohort 2

\footnotetext{
- CV features only

- CTD features only
}

Cohort 3

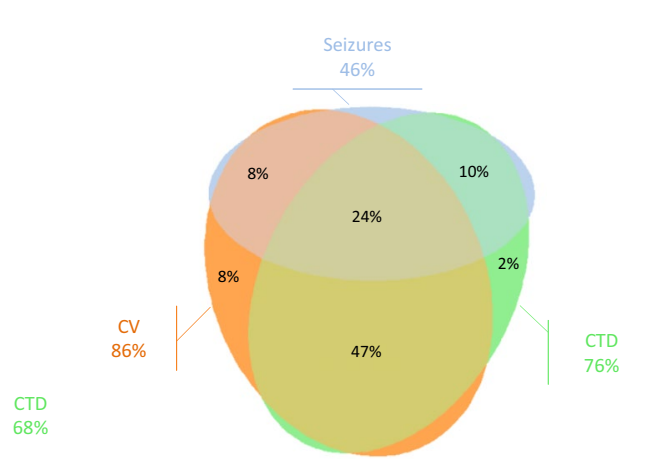

Fig. 1 (See legend on previous page.) 


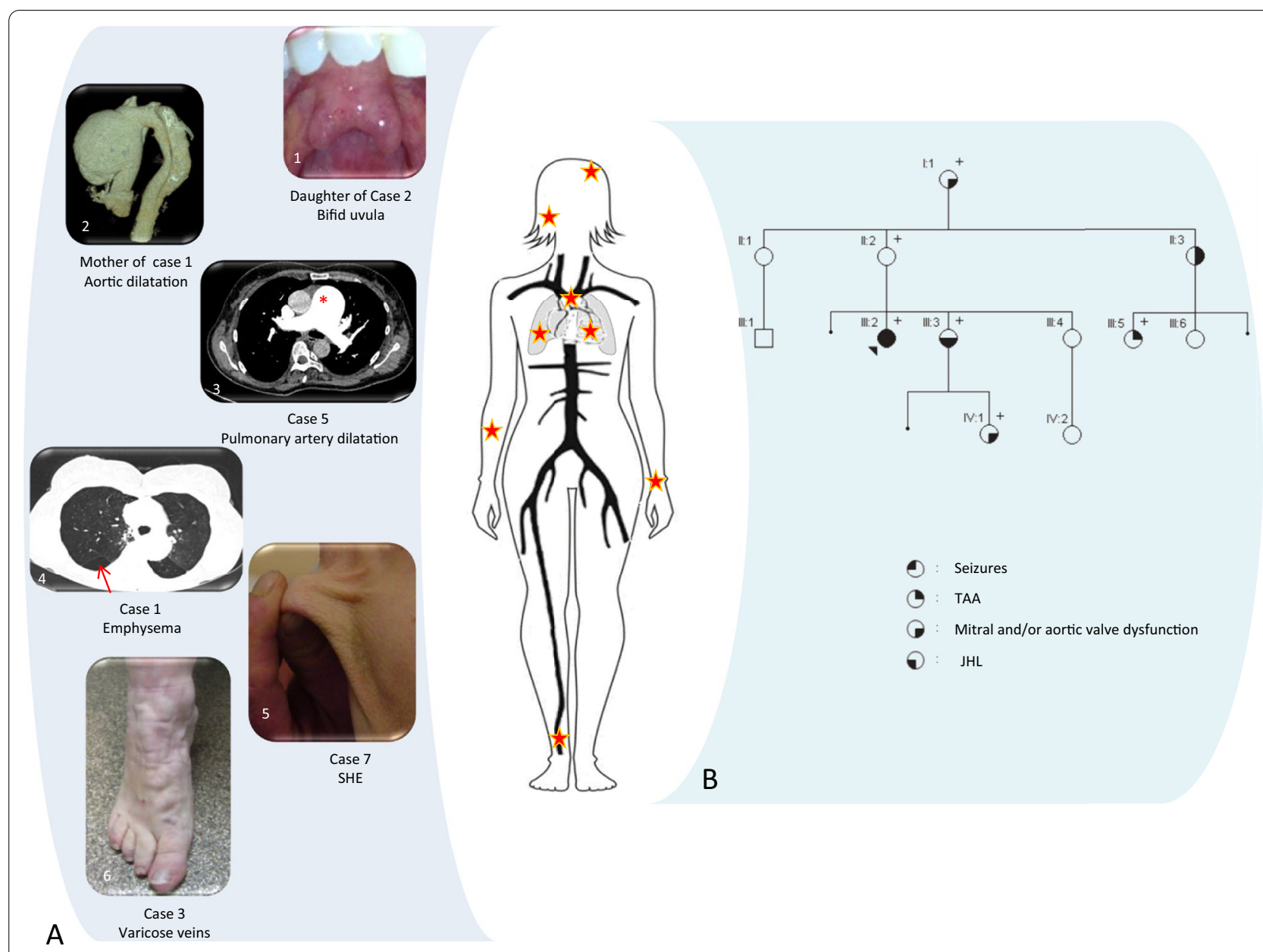

Fig. 2 Clinical characterization of cohort 1. A Inter-individual phenotypic variability: Schematic representation of features in the whole body. $1 /$ Bifid uvula in daughter of index-case\#2. Her phenotype is similar to her mothers', including seizures, and PNH. Angio-CT scan demonstrated dilation of both the ascending aorta $(43 \mathrm{~mm}$ ) and the pulmonary artery without arterial tortuosity. 2/ Mother of index-case\#1 had an unexpected $140 \mathrm{~mm}$-diameter TAAA (associated to a bicuspid aortic valve not shown). She died at 62 from dissection. 3/ Pulmonary artery dilatation (55 mm) in index-case \#5. She was referred for idiopathic Pulmonary arterial Hypertension (PAH) associated with syndromic aortopathy. CV examination revealed major dilatation of pulmonary artery $(55 \mathrm{~mm})$, aortic dilatation at the sinus of Valsalva $(52 \mathrm{~mm})$ and a mild mitral valvulopathy. 4/ Emphysema of unknown origin in index-case \#1: She presented a TAAA, JHL, a minor scoliosis, easy bruises and enlarged scars. 5/ SHE in index-case\#7: Clinical examination revealed some CTD features: JHL with major hypermobility (Beighton score 9/9), kyphoscoliosis, thin and translucent skin, spontaneous bruising and atrophic scars along with acrogeria and moderate arachnodactyly. 6/ Severe varicose veins due to chronic venous insufficiency in the foot of index-case\#3. She was operated for TAAA and had pulmonary artery dilation with PAH. Clinical examination found velvety skin without SHE, JHL (Beighton score 5/9) and scoliosis along with disorders of the temporomandibular joint. B Intra-famillial phenotypic variability. Family 9 pedigree. Index-case \#9 (III-2), 30 year-old, had an aortic aneurysm (Z-score at Valsalva Sinus =2.44), a leaky aortic valve and JHL. Cerebral MRI previously performed for seizures had revealed PNH. Molecular analysis identified an FLNA pathogenic LoF variant c.6772G>T, p.(Glu2258*).Clinical evaluation of the mother (II-2) did not reveal neurological symptom, neither JHL nor SHE. CV evaluation wasn't available. The sister (III-3) had JHL and an aortic valve insufficiency without aortic aneurysm nor seizures. The cousin (III-5) had aortic dilation (Z-score at Valsalva Sinus = 2.4), normal aortic valve function, and absence of JHL and seizures. Her mother (II-3) had a mitral valve insufficiency and an aortic dilatation (Z-score at Valsalva Sinus $=3.5$ ) without CTD features or seizures. The grand-mother (I-1) had aortic valve dysfunction, but her complete clinical evaluation was not available. In this pedigree, none of the six relatives carrying the FLNA pathogenic variant had neurological symptoms

There was a median of $2 \mathrm{CV}$ anomalies [min-max: $0-7]$ and 1 CTD feature [min-max: 0-3] in these patients. In total, $77 \%(n=17 / 22)$ of patients presented CV anomalies (Fig. 1A, B, C).The most prominent CV features were aortic aneurysm in $67 \%$ of patients $(10 / 15)$ with aortic insufficiency in $50 \%$ of patients $(10 / 20)$, followed by bicuspid aortic valve in $29 \%(5 / 17)$. We did not have the information about $\mathrm{CV}$ features in one patient.

CTD features were described in 68\% (15/22) of patients (Fig. 1C, Additional file 2: Data S2). The most prevalent 
features were the two major criteria for CTD, JHL in 53\% $(9 / 17)$ and SHE in 33\% (5/15). We did not have the information for any CTD features in one patient.

Clinical presentation was highly variable (Fig. 1A, B) with the combination of seizures and $\mathrm{CV}$ anomalies and CTD features in $29 \%(6 / 21)$ of patients, seizures and CV anomalies in 24\% (5/21) and CV anomalies and CTD features without seizures in 43\% (9/21) (Fig. 1C).

\section{Literature patients}

We performed a rapid literature review following a multistep process in order to build a third cohort with at least one CTD and/or CV feature from the literature (Fig. 3, Additional file 5: Method S2, Additional file 4: Data S4). After step 1 and 2 (Additional file 5: Method S2) we identified 61 cases $(25 \%)$ as having unambiguous $\mathrm{CV}$ and/or CTD features among 245 index-cases [1-6, 9-11, 14-36].

In step 3 of our literature review, all FLNA variants were reclassified using HGVS and ACMG classification [13] (Fig. 3, Additional file 4: Data S4, Additional file 5: Method S2). We excluded patient \#55 with unspecified variant [16]. A total of 62 variants in 60 patients were identified. After applying ACMG criteria, 48 variants were classified Pathogenic (Class 5), 10 Likely Pathogenic (Class 4). Two variants were reclassified of Unknown Significance (Class 3) in the absence of the author's argument to classify the variant. We excluded patient \#33 for which this two variants were reclassified to Likely Benign [15].

Detailed phenotypic description of these 59 indexcases is available in Table 1 and Additional file 4: Data S4. In this cohort, $66 \%$ were females. The median age was 15 years [min-max: $0-71]$.

PNH was proven in $93 \%$ of patients $(n=55), 46 \%$ $(\mathrm{n}=26 / 56)$ had seizures and $31 \%(\mathrm{n}=14 / 45)$ had mega cisterna magna (information on both signs was not available for three patients). One male patient (1.6\%) did not have PNH but presented with CTD and CV signs. Information was unavailable about cerebral MRI for 5\% $(\mathrm{n}=3)$ of patients, all presenting with CV and CTD signs. Patients had a median of $2 \mathrm{CV}$ anomalies [min-max: 1-8] and 1 CT feature [min-max: 0-3].

In total, $86 \%(\mathrm{n}=51)$ patients presented $\mathrm{CV}$ anomalies (Fig. 1A, C, Additional file 2: Data S2). The most frequent anomalies were aortic aneurysm in $51 \%(\mathrm{n}=27 / 53)$, followed by PDA in $41 \%(n=21 / 51)$ and mitral valve anomaly in $41 \%(n=19 / 46)$. Cardiovascular information was not available or incomplete for $14 \%(n=8)$ of subjects. In this group, males were present, and when looking for gender differences we identified an excess of tricuspid valve anomalies (prolapse or dysplasia) in males $(p<0.0001$, Additional file 5: Table S1). We did not find difference in neurological features between males and females.

CTD features were described in $76 \%$ of patients $(\mathrm{n}=45)$. Regarding major CTD criteria, $73 \%$ had JHL $(n=29 / 40)$ and $42 \%$ had SHE $(n=14 / 33)$. When reported, SHE was always associated with JHL and $10 \%$ $(n=6)$ had none of them. One patient did not present CTD features (patient \#3). Information about all CTD features was unavailable in $10 \%(n=6)$ of patients.

According to our literature search criteria, all patients had at least one CV or CTD feature. Overall, $86 \%(n=51)$ of the patients had at least one CV sign and $76 \%(n=45)$ had at least one CTD feature as previously defined (Fig. 1A), and $75 \%(n=35)$ had both types of signs. In total, $47 \%$ of patients $(n=23)$ had a phenotype combining CTD and CV features without neurological symptom.

\section{Global analysis of PVNH1 patients with CV and CTD features}

In order to perform a global analysis of clinical features in all patients, we sought for possible bias or specificities for patient's selection by comparing the prevalence of each feature between the three cohorts (Additional file 5: Table S2).

We first compared cohort 1, mainly corresponding to an enrolment via the Reference Center for Rare Vascular Diseases, to the two other groups. The age at diagnosis was significantly higher in cohort 1 (median age $=38$ years, mean age $=40$ years) than in the two other cohorts (median age $=15$ years, mean age $=19$ years, $p<0.001$ ). We did not detect any difference in the prevalence of other features (Additional file 5: Table S2). We then compared cohort 2 and literature patients, considering that the selection procedure was similar in these two groups. We excluded all features that were significantly different in the subsequent global analysis.

The analysis revealed that more than half of patients did not have seizures $(n=47 / 89)$, and that aortic aneurysm was present in $58 \%$ of patients $(n=46 / 78)$. The other remarkably frequent features were PDA in $34 \%$ of patients $(n=24 / 70)$, VSD/ASD in $29 \%$ of patients $(\mathrm{n}=17 / 59)$, JHL in $67 \%$ of patients $(\mathrm{n}=45 / 67)$ and SHE in $40 \%$ of patients $(n=23 / 57)$.

\section{Variants type and distribution in PVNH1 patients with CV and CTD features}

We gathered, analyzed, classified or reclassified a total of 93 FLNA variants in 92 patients with CV and/or or CTD features (Fig. 4), of which 90 could be classified as pathogenic or likely pathogenic and three as VUS (Additional file 1: Data S1, Additional file 3: Data S3 and Additional file 4: Data S4). 


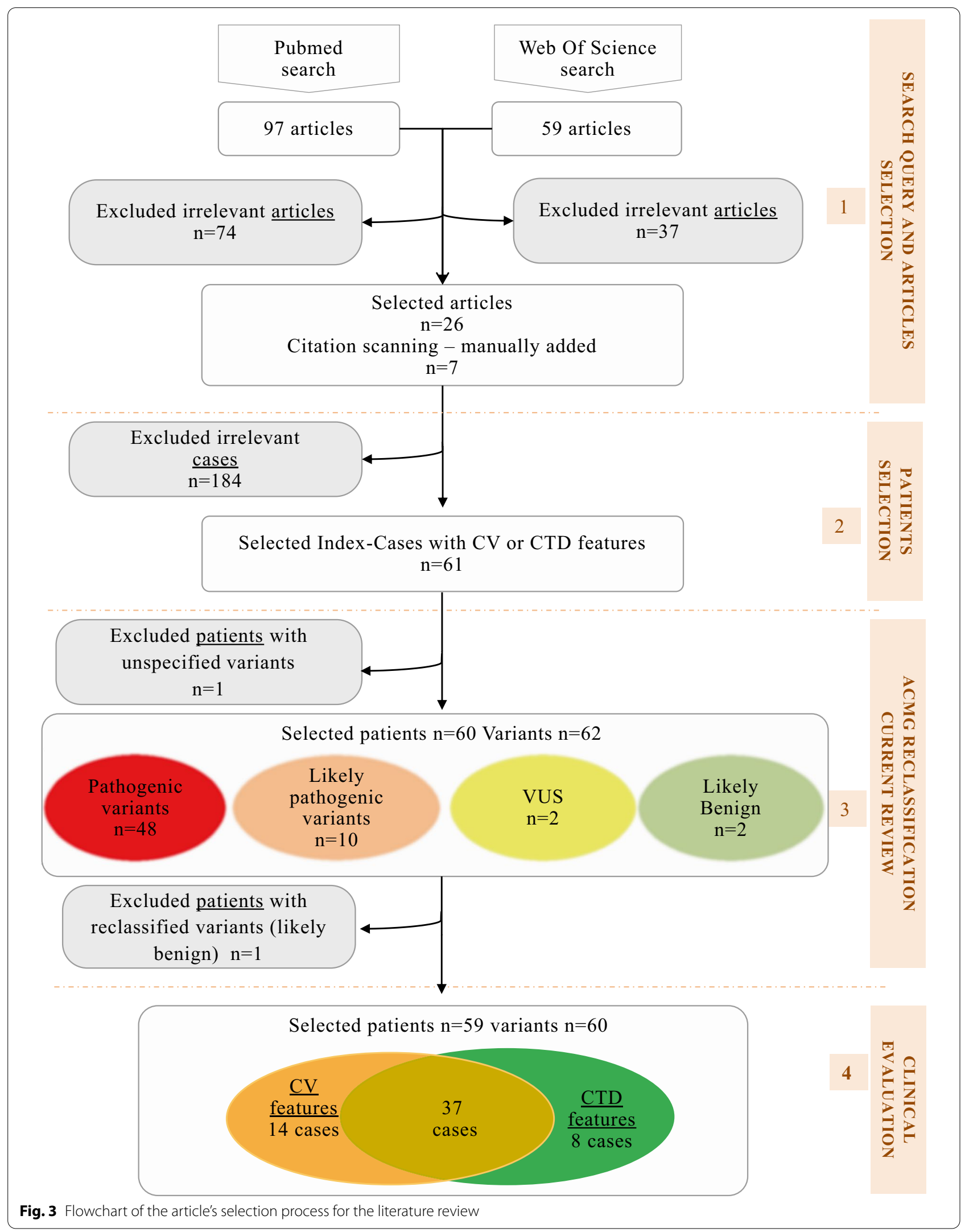




\section{Cohort 1}

c. 596del; p.(Gly199Alafs*43)
c. 705G A P. (Irp235Ter)

$c .623-16>A, p$ ? c.11

$1120 \_1125$ delinsTCTTG, p.val374SerfsTer2) c.1111del, p.(Glu371Argfster14)

c. $1828+1 \mathrm{G}>\mathrm{T}$,

p.(Ser564ArgfsTer11)

c.2625G>A, p.Lys876SerfsTer11

c.3677 3684del, p.(Pro1226LeufsTer38 c. 4143-12T>A, p? c.4143-2del, p.?

c.4501_4502del, p.(Asn1501GInfsTer3)

c. $4543 \mathrm{C}>\mathrm{T}$, p.(Arg1515Ter)

c.4812_4822delinsCCAG, p.(Thr1605GInfsTer6)

$$
\text { c.4828_4838del, p.Pro1610ArgfsTer12 }
$$

c.5184C $>$, p.(Gly1728Gly)

c.5336del, p.Gly1779ValfsTer7

c.5417-1G>T p.?

c.6087_6097del, p.(His2029GInfsTer32) c.6204del, p.(Phe2086LeufsTer65) c.6230 6231dup, p.Gly2078MetfsTer56

c.6772G>T, P.(Glu2258Ter)

$\begin{array}{ll}\text { (i) } & \text { g.(? 153599614) (153595764 153595219)del } \\ \text { (ii) } & \text { g.(153585618_153583441)_(153577216_?)del } \\ \text { (iii) g.(153594391_153593855)_(153577216_?)del } \\ \text { (iv) g.(?_153599730)_(153592893_153592534)del } \\ \text { (v) g.(?_153599614)_(153577216_?)del }\end{array}$

(ii)*2 (i)
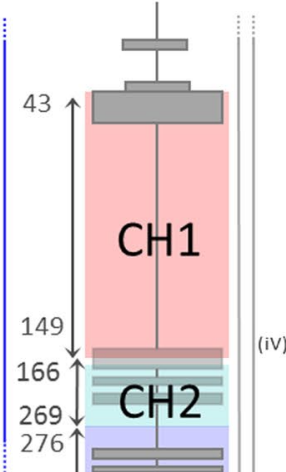

c.62_64dup, p.(Val21dup)

c. $116 \mathrm{C}>A$, p.(Ala39Glu); c.116C>G, p.(Ala39Gly)

c.220G >A, p.(Gly74Arg)

c. $238 \mathrm{C}>\mathrm{G}$, p. (Leu80Val)

c.304A>G, p.(Met102Val)

c.356T>A, p.(Ile119Asn);

c.365T>G, p.(Val122Gly)

c. $373+1 \mathrm{G}>\mathrm{A}$, p.?

c.381G>C, p.(Lys127Asn)

c.38C

(iv) c. $446 \mathrm{C}>\mathrm{T}$, p.(Ser149Phe)

c. $720+2 T>C, p . ?$

c.853C $>$ T, p. (Arg285Cys) X2

c.885_892del, p.(Asn296GlufsTer38), p.0

c.994del, p.(Val332Ter)

c.1065G>A, p.(Lys355Lys)

c.1621G>T, p.(Glu541Ter)

c.1692-2 A>G, p.Ser564ArgfsTer11

c.1790_1791del, p.(Phe597CysfsTer23)

c.1828+2T>G, p.?
c. $1829-2 A>G, p . ?$

c.1829-1G>C, p.?

c. $2002 \mathrm{C}>\mathrm{T}, \mathrm{p} . \mathrm{ln} 668 \mathrm{~T}$

c.2193C>G, p.(Tyr731Ter) ; c.2193C>A, p.(Tyr731Ter)

\section{ROD1}

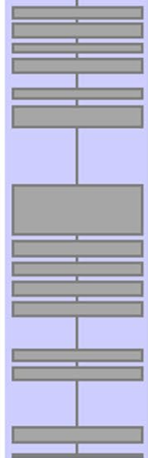

1740

$1779 \uparrow$
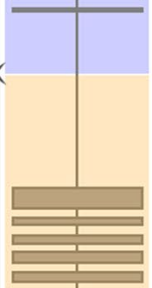

c. $2762 \mathrm{del}, \mathrm{p}$.(Arg921GInfsTer25)

\section{c. $2807 \mathrm{~A}>$ G p. (Lys936Arg)}

c.3045_3049del,p.(Val1016LeufsTer10)

c.3153dup, p.(Val1052ArgfsTer17)

c.3582del, p.(Ile1195LeufsTer91)

c. $3742 \mathrm{C}>\mathrm{T}, \mathrm{p} .(\mathrm{G} \ln 1248 \mathrm{Ter})$

(V) c.4039del, p.(Val1347CysfsTer3)

c.4147del, p.(Ala1383LeufsTer10)

c.4303+4_4303+11del, p.(?)

c.4446_4447dup, p.(Leu1483TyrfsTer19)

c.4574dup, p.(Tyr1525Ter)

c.4596dup, p.(Ser1533GlufsTer12)

c.4617_4618del, p.(Leu1540AlafsTer4)

c.5314G $>$ A,p.(Ala1772Thr)

c.5498_5504delinsAC, p.(Ala1833AspfsTer3)

c.6425 6428del, p.[Glu2142AlafsTer22,Gly2127_Pro2167del] c.6586dup, p.(His2196ProfsTer11)

c.6635_6638del, p.(Val2212AlafsTer2)

c.6769G >C, p.(A)

(ii) $\mathrm{c} .6915 \mathrm{C}>\mathrm{G}, \mathrm{p}$.(Tyr2305Ter)

I

2516

ROD2

c. $7045 \mathrm{C}>\mathrm{T}, \mathrm{p} .(\mathrm{G} \ln 2349 \mathrm{Ter})$

c. $7153 \mathrm{C}>\mathrm{T}, \mathrm{p} .(\mathrm{G} \ln 2385 \mathrm{Ter})$

\section{c.7813del, p. (Leu2605Trpfs*2)
c.7896 G>A, p.(Trp2632Ter)}

c. [7921C>G;7923del], p.[(Pro2641Ala;Tyr2642ThrfsTer63)]

Fig. 4 Variant type and distribution in PVNH1 patients with CV and CTD features. Schematic representation of the FLNA domains and overview of FLNA variations from PVNH1 patients with CV and CTD features (In blue: Cohort 1, in Red: Cohort 2, in grey: Literature patients). Variations with proven somatic mosaicism are underlined. Copy Number Variants are indicated with brackets, from (i) to (v) (see the bottom-left sidebar) 
The most frequent variant types were frameshift $(n=34)$ and missense $(n=20)$ variants, of which one was a VUS (Additional file 5: Table S3). Large deletions, intronic, synonymous, and in-frame indel variants were rare. Amongst patients for which inheritance was known, $43 \%(n=21 / 49)$ were proven de novo, and two were germline mosaicism. Information on inheritance was not available for 43 patients.

Variant distribution was globally homogeneous throughout the gene (Fig. 4). Considering that only missense variants did not eventually lead to nonsensemediated mRNA decay and could have an impact on the protein structure or function, we studied the distribution of the 19 non-VUS missense variants within the different domains of FLNA regarding their relative size: N-terminal, $\mathrm{CH} 1, \mathrm{CH} 2$, ROD1, Hinge 1, ROD2 and C-terminal. Two domains showed significant enrichment in variant distribution regarding their relative size: the $\mathrm{CH} 1$ domain was highly enriched with almost two thirds of variants $(\mathrm{n}=12, p=2.289 \mathrm{E}-8)$ and the ROD1 domain with $5 \%$ of variants $(\mathrm{n}=1, p=0.0156)$. The other domains (N-terminal, $\mathrm{CH} 2$, Hinge 1, ROD2 and C-terminal) were not significantly enriched in pathogenic missense variants (Additional file 5: Table S4).

\section{Discussion}

We performed a systematic analysis of some neurological and non-neurological features in patients suffering from PVNH1 caused by a FLNA loss of function variant. Our aim was to focus on CV and CTD features, and to delineate as precisely as possible their spectrum in PVNH1, independently of clinical or radiological $\mathrm{PNH}$.

\section{Frequency of neurological features, age at diagnosis, and diagnostic delay}

On the whole, we have observed that half of PVNH1 patients with CV and/or CTD features do not have any neurological symptom: although all but one patients included in this study presented $\mathrm{PNH}$, only $50 \%$ $(n=5 / 10)$, and $46 \%(n=26 / 56)$ of patients from cohort 1 and literature had seizures, respectively. In this latter group, this point should be taken with caution as patients without seizure were younger (mean 10.73 years, minmax: 0-71) than the reported mean age of onset of seizures (mean 15.75 years [1]). In contrast, the prevalence of this sign in cohort $2(70 \%, n=16 / 23)$ was highly consistent with published data [1]. Our study reinforces the knowledge of variability of neurological symptoms in this condition, and points to the associated risk of misdiagnosis and diagnostic delay. The description of patient \#9 pedigree in cohort 1 is highly illustrative of this inconsistency: among six affected cases in this family only one presented with seizures, the index case who allowed definitive diagnosis in her relatives. Consistent with this finding, when evaluating age at diagnosis, we brought to light important differences among cohorts: median age at diagnosis in cohort 1 was 38 years old, compared to 16 and 15 years old respectively for the two other groups. We believe that this difference is the consequence of long diagnostic delay in cohort 1 patients, for various reasons, including the low prevalence of neurological symptoms in these patients.

\section{Diversity of CV and CTD features, and various syndromic presentations}

We have shown that clinical presentations are highly variable among patients as well as among families, with a wide range of possible combinations in each patient (Figs. 1, 2). This observation is in line with other CTD and rare vascular disorders, where highly heterogeneous and inconsistent presentations amongst individuals are the rule.

The five most prominent features remained TAAA and JHL in more than half of patients, PDA and SHE in close to one third of patients, and VSD/ASD. A previous study focusing on $\mathrm{CV}$ features in PVNH1 patients showed that more than $40 \%$ of these individuals had CV features [14], mainly ascending aortic aneurysm (18\%) and PDA (23\%). These findings are partially consistent with our data as well as with other publications $[9,10]$. However, these frequencies have limited comparability because of important differences in patient selection like inclusion of patients with documented FLNA-related $\mathrm{PNH}$ and absence of FLNA variants reclassification, potentially introducing a bias in the interpretation of clinical data.

Emphysema was recently identified [17, 29] as a complication of PVNH1. In our study, we specifically investigated this feature because severe emphysema in patient \#1 from cohort 1 was particularly remarkable. On the whole, emphysema was found in 24 out of 57 patients included in our study, including 4 out of 10 patients in cohort 1, and more than half in literature patients (Table 1 and Additional file 5: Table S2). Recent retrospective study in a tertiary children hospital identified 6 in 9 FLNA LoF patients with various respiratory or lung imaging anomalies, including emphysema [37]. These observations led us to consider that this complication is still underestimated in FLNA LoF patients, and should be systematically investigated.

\section{FLNA molecular diagnosis}

We provided a systematic analysis and reclassification of FLNA genetic variants in this study, and showed that the FLNA CH1 domain is significantly enriched in missense pathogenic variants. This domain was already known to be enriched in any type of pathogenic variant beyond 
missense variants in PVNH1 patients, whatever the clinical presentation [1]. Our analysis involved only missense variants, and confirms that the $\mathrm{CH} 1$ domain and its pathogenic variants are critical for FLNA function. We also show that the $\mathrm{CH} 1$ domain is not uniquely associated with the onset of neurological features, but in various aspects of PVNH1, including CV and CTD features. However, in light of the absence of missense variants in cohort 1 , we cannot exclude that LoF variants other than missense would be associated with severe cardiovascular events. Further observations in a larger group of patients would help refining this hypothesis.

This study demonstrates a high level of de novo variants, which is another clue for a possible diagnostic delay. We identified as much as $43 \%$ patients with proven de novo variants, which imply that family history is frequently uninformative. Altogether, these facts illustrate the need to shift from a neurological-centered diagnostic process for patients harboring an FLNA LoF variant to a more interdisciplinary approach in order to identify these patients accurately and earlier. It could be relevant to consider this disorder as an "FLNA deficiency syndrome", in place of PVNH1 which does not reflect the entirety and complexity of this condition. Systematically including FLNA in NGS-based CTD and CV diagnostic panels might prospectively confirm our results and refine the involvement of FLNA LoF variants in rare vascular and connective tissue disorders. These findings imply that physicians facing patients with CTD and CV features need to be aware of a possible FLNA-related disorder despite negative history of seizures. As a consequence, brain MRI should be systematically performed to diagnose PNH when an FLNA LoF variant is suspected or identified in a patient with CV or CTD features regardless of neurological symptoms.

\section{Limitations}

First, the patients described in cohort 1 were referred to our Reference Center because they had a history of $\mathrm{CV}$ anomalies. We consider that this group is probably clinically different from cohort 2 and literature patients, because they were not recruited for neurological signs. However, the small size of this cohort did not allow us to confirm further this opinion.

Second, we built cohort 2 by selecting registry patients for whom we had information on CV or CTD signs. This information was unavailable for 88 among 111 patients, leading to a possible underestimation of the prevalence of $\mathrm{CV}$ complications and EDS features.

Third, Cohort 3 is also affected by possible bias. Our literature review did not follow the criteria of a systematic review. Although we followed a strict protocol for studies inclusion, exclusion, and data extraction, we did not perform any quality assessment of the selected studies. Another point relates to the criteria for article selection of PVNH1 patients with at least one CV or EDS feature. It is noticeable that we excluded 108 patients [2, 14] in the literature review because individual clinical description was not available. Moreover, the majority of patients reported in the literature may not have benefited of expert cutaneous, articular and arterial assessment, possibly underestimating the prevalence of $\mathrm{CV}$ complications and EDS features. The prevalence of these features in patients recruited for neurological signs can be only roughly estimated with our approach. A precise evaluation in a retrospective context would need clinical reexamination of all carriers of a Lof $F L N A$ variant.

\section{Conclusion}

Our study confirms that associated and entangled CV anomalies and EDS features are frequent and are a critical component of the clinical diagnosis of PVNH1.The presence of CTD and/or CV features without neurological symptoms is not uncommon and should not be overlooked in considering a diagnosis of PVNH1. Owing to the prevalence and severity of aortic disease in this condition, CV surveillance should be performed systematically in PVNH1 patients.

The association of JHL with or without SHE and cardiac valve anomalies and/or thoracic aorta dissection/ aneurysm in the absence of seizures must trigger FLNA molecular analysis similarly to other CTD genes. A brain MRI can be performed for searching PVNH to support the molecular diagnosis. Despite X-linked dominant inheritance, our data suggest that evocative phenotype in male patients should not exclude the search for a FLNA variant.

\section{Patients and methods Cohort 1 \\ Patients}

The patients herein described were recruited because of CV anomalies and CTD features from 2014 to 2020 during outpatient visits in the French Reference Center for Rare Vascular diseases (Coordinating Center: HEGP, Paris, France; Collaborating Centers: Departments of Vascular Medicine of Nancy University Hospital; Internal Medicine of Clermont-Ferrand University Hospital; Pneumology of Kremlin-Bicêtre University Hospital; and Medical Genetics of Marseille, Nice, Rennes and Toulouse University Hospitals). Owing to the specificities of patients recruitment in our Centre, we did not recruit any patient because of neurological signs.

Medical information including family history and clinical findings was collected using standardized questionnaire (Additional file 5: Method S3). A total of 32 
physical signs or anomalies were gathered, including neurological, CV, skin, articular and gastrointestinal features frequently found in CTD and rare vascular diseases. Physical examination was performed by a panel of clinicians specialized in CV diseases and CTD and reviewed by expert clinicians from the Coordinating Center. Clinical diagnostic criteria were ascertained as per the 2017 EDS international classification [12]. Thoracic aorta aneurysm was defined as a Z-score above 2 as described by Campens [38]. Brain MRI preceded the molecular diagnosis in four patients (patients 2, 4, 8 and 9) and was performed following molecular diagnosis in four patients (patient 1, 3, 5, 6). Blood samples were obtained from relatives whenever possible.

\section{Genetic analysis}

Molecular diagnosis was performed in the Genetics Department of The French Reference Center for Rare Vascular Diseases (Coordinating Center, HEGP, Paris, France), by a gene panel approach dedicated to CTD and aortopathies using Next Generation Sequencing (Additional file 5: Method S4). Patients with a FLNA variant of interest were selected for constitution of this cohort (variant of unknown significance, likely pathogenic and pathogenic variants according to HGVS/ACMG classification) [13]. All patients with FLNA variants were included in this cohort.

\section{Cohort 2}

This cohort was built from the PVNH1 registry held by the National Reference Diagnostic lab for filaminopathies A at Bordeaux University Hospital. This registry includes patients with FLNA LoF variants, analyzed from 2005 to 2020 ( $n=111$ index-cases), mainly recruited through neurological symptoms, or PVNH discovered on brain MRI at any age.

Molecular analyses of these patients are detailed in Additional file 5: Method S1. We extracted from this registry patients with at least one CTD or CV anomaly whose medical records were retrospectively reviewed using the same questionnaire as in cohort 1 .

\section{Literature patients}

\section{Literature review and patients' evaluation process}

A rapid review of the literature was conducted to (1) identify precisely and unambiguously $\mathrm{PVNH} 1$ published cases presenting with at least one CV and/or CTD feature (2) confirm the pathogenicity of FLNA variants in these patients, according to the most recent HGVS/ ACMG criteria and (3) precisely evaluate the prevalence of each CTD and CV feature in these patients. The flowchart of this process is detailed in Fig. 3. The complete procedure of articles, patients, and variants selection is detailed in Additional file 5: Method S2.

Delineation of the recurrent CTD and CV features in PVNH1 A first global analysis of cohorts 1,2 and published cases led to the identification of 29 features of which 10 CTD and $12 \mathrm{CV}$ anomalies were found once or more. They were completed by the three neurological features mentioned in the questionnaire (seizures, $\mathrm{PNH}$ and mega cisterna magna). The 7 remaining features were removed from further analyses. The by-cohort analysis was then led on this subset of 25 features.

\section{Statistical analysis}

Data were analyzed using Excel 2016 software (Microsoft $^{\circledR}$ ) and RStudio 2017 software (RStudio, Inc.). Data are presented as percentages and numbers for qualitative variables and as median and extreme values for continuous variables. Percentages were calculated after excluding patients for which the data were unknown. To analyze patients' characteristics between the three cohorts, time-independent qualitative variables were compared through logistic regression. Chi-squared test or Fisher's exact test (whenever expected frequencies were $<5$ ) were performed to compare variables according to sex. Fisher's exact test of independence was also performed to compare the distribution of variants in specific domains, defined as the ratio of mutated residues over all residues in each domain. Age was the only quantitative variable and it was studied using Student's test. All $p$ values were two-sided and considered significant if $<0.05$.

\section{Supplementary Information}

The online version contains supplementary material available at https://doi. org/10.1186/s13023-021-02128-1.

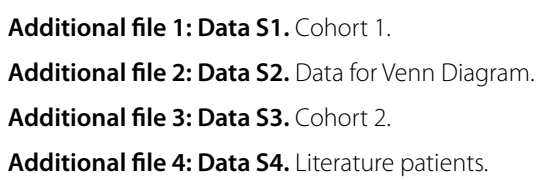

\section{Acknowledgements}

We thank all patients and their families for their kind collaborations. We acknowledge the contributions of all clinicians. We also thank the technicians of the genetics laboratory for their contribution to the FLNA genetic analysis. 
We thank Caroline Truntzer for participation to the pictures elaboration. As this article is not categorized as human research, the local ethics review committee neither reviewed nor approved the publication of these cohorts. Acceptable anonymity was achieved, and HIPAA identifiers are not available in the manuscript.

\section{Bordeaux-cohort collaborators}

Dr. Anne Dieux ${ }^{23}$, Dr. Fabien Labombarda ${ }^{24}$, Dr. Sylvain RHEIMS ${ }^{25}$, Dr. Odile Boute $^{23}$, Dr. André Vincentelli ${ }^{26}$, Pr. Annick Toutain ${ }^{27}$, Pr. Sylvie Odent ${ }^{19}, \operatorname{Pr}$. Gaetan Lesca ${ }^{28}$, Dr. Marie Vincent ${ }^{29}$, Dr. Juliette Piard ${ }^{30}$, Maud Favier ${ }^{30}$, Pr. $^{2}$ Philippe Derambure ${ }^{31}$, Pr. Patrick Edery ${ }^{28}$, Dr. Susanne Thummler ${ }^{32}$, Dr. Marion Gérard $^{33}$, Dr. Fanny Morice Picard ${ }^{34}$, Valérie Layet ${ }^{35}$, Cécile Laroche ${ }^{36}$, Dr. Laurent Pasquier ${ }^{19}$, Dr. Elisabeth Sarrazin ${ }^{37}$, Pr. Thierry Billette de Villemeur ${ }^{38}$, Dr. Lucie Guyant-Marechal ${ }^{39}$.

23. Pôle de Biologie Pathologie Génétique, Hôpital Jeanne de flandre, Lille. 24. Service de cardiologie, CHU de Caen, Hopital Cote de Nacre, Caen, France. 25. Department of Functional Neurology and Epileptology, Hospices Civils de Lyon and University of Lyon, Lyon, France, Lyon's Neurosciences Research Center (INSERM U1028/CNRS UMR5292), Lyon, France, Member of the European Reference Network EpiCARE. 26. Service de Chirurgie Cardiaque, CHU Lille, Lille, France. 27. UMR 1253, iBrain, Université de Tours, Inserm, 37032 Tours, France; Service de Génétique, Centre Hospitalier Universitaire, 37044 Tours, France. 28. Department of Genetics and Reference Center for Developmental Disorders, Lyon University Hospital, Groupement Hospitalier Est, Hospices Civils de Lyon, Bron, France. 29 Service de Génétique Médicale, CHU Nantes, 9 quai Moncousu, 44093 Nantes Cedex 1, France; L'Institut du Thorax, INSERM, CNRS, Université de Nantes, 44007 Nantes, France. 30. Centre de Génétique Humaine, Centre Hospitalier Régional Universitaire, Université de Franche-Comté, Besançon, France. 31. Neurophysiologie clinique, CHU de Lille, Hôpital Roger Salengro, F-59037, Lille cedex, France. 32. Service de Psychiatrie de l'Enfant et de l'Adolescent, Hôpital de Nice CHU-Lenval, Nice, France. 33. Department of Genetics, Normandy Center for Genomic and Personalized Medicine, Caen University Hospital, Caen, France. 34. Service de Dermatologie, CHU de Bordeaux, Bordeaux, France. 35. Consultations de génétique, Groupe Hospitalier du Havre, Le Havre, France. 36. Département de pédiatrie médicale CHU de Limoges - Hôpital de la mère et de l'enfant. 37. Reference Center of Neuromuscular Rare Diseases, CHU Fort de France, Pierre Zobda Quitman Hospital, 97200 Martinique, France. 38. Service de Neuropédiatrie - Pathologie du développement, Centre de référence des déficits intellectuels de causes rares, Sorbonne Université, UPMC, GRC ConCer-LD and AP-HP, Hôpital Trousseau, Paris, France. 39. Genetique Clinique, Hopital Charles Nicolle, Rouen, France.

\section{Authors' contributions}

Data curation: CB, XJ, JA; Formal analysis: CB, SA, JA; Investigation: CB, SA, JA, NHP, MF, LC, SZ, KB, LS, ZKK, A-CB, LB, TB, MF, CQ, BC, DW, PF, CG, TM; Bordeauxcohorts collaborators; Methodology: JA, Supervision: $X J$, JA; Writing-original draft: CB, SA; Writing - review and editing: SA, JA, NHP, XJ, AL. All authors read and approved the final manuscript.

\section{Funding}

We do not have funding to declare.

\section{Availability of data and materials}

All data generated or analyzed during this study are included in this published article and its supplementary information files.

\section{Declarations}

\section{Ethics approval and consent to participate}

Written informed consent was obtained for all patients including permis sion to scientific publication and pictures. For child participants, consent was obtained from the parent or guardian. Genetic testing was performed in accordance with French legislation regarding genetics diagnostics tests (French bioethics law 2004-800). As this article is not categorized as human research, the local ethics review committee neither reviewed nor approved the publication of these cohorts. Acceptable anonymity was achieved, and HIPAA identifiers are not available in the manuscript.

\section{Competing interests}

The authors declare that they have no competing interests.

\section{Author details}

${ }^{1}$ Département de génétique, Centre national de référence pour les maladies vasculaires rares, centre de référence européen VASCERN MSA, Hôpital Européen Georges Pompidou, AP-HP, 20 rue Leblanc, 75015 Paris, France. ${ }^{2}$ INSERM, U970 PARCC, Université de Paris, Paris, France. ${ }^{3}$ Service de Médecine Vasculaire, Hôpital Saint Eloi, CHU Montpellier, Montpellier, France. ${ }^{4}$ Département de génétique médicale, Centre national de référence pour les maladies rares Neurogénétiques, Hôpital Pellegrin, CHU Bordeaux, Bordeaux, France. ${ }^{5}$ Laboratoire de maladies rares: Génétique et Metabolisme (MRGM), INSERM U1211, Université de Bordeaux, Bordeaux, France. ${ }^{6}$ Faculté de médecine, Université de la Sorbonne, Paris, France. ${ }^{7}$ Service de chirurgie vasculaire et endovasculaire, Centre aortique tertiaire, Hôpital universitaire Pitié-Salpêtrière, AP-HP, Paris, France. ${ }^{8}$ Inserm UMRS 1116 DCAC, Université de Lorraine, Nancy, France. ${ }^{9}$ Division de médecine vasculaire et centre de compétence régional pour les maladies vasculaires rares et autoimmunes systémiques, Centre Hospitalier Régional Universitaire de Nancy, Nancy, France. ${ }^{10}$ Centre de Référence des Syndromes d'Ehlers-Danlos non Vasculaires, Hôpital Raymond Poincaré, Assistance Publique Hôpitaux de Paris, Garches, France. ${ }^{11}$ UMR U1179 INSERM, Université Versailles Saint-Quentin, Montigny-le-Bretonneux, France. ${ }^{12}$ Université ParisSaclay, Le Kremlin Bicêtre, France. ${ }^{13}$ UMR_S 999, INSERM, Groupe hospitalier Marie-Lannelongue -Saint Joseph, Université Paris-Sud, Le Plessis-Robinson, France. ${ }^{14}$ Service de Pneumologie, Hôpital Bicêtre, APHP, Le Kremlin-Bicêtre, France. ${ }^{15}$ Unité de génétique médicale 2, Hôpital L’Archet, Nice, France. ${ }^{16}$ INSERM U1245, Normandy center for Genomic and Personalized Medicine, Normandie Univ, CHU Rouen, 76000 Rouen, France. ${ }^{17}$ Centre de référence régional Marfan et apparentés, Centre aortique, Hôpital La Timone, AP-HM Marseille, France. ${ }^{18}$ Département de Génétique Médicale, Hôpital La Timone, CHU de Marseille, Marseille, France. ${ }^{19}$ Service de Génétique Clinique, Centre de Référence Maladies Rares CLAD-Ouest, ERN ITHACA, CHU Rennes, Hôpital Sud, Rennes, France. ${ }^{20}$ Service de génétique médicale, Hôpital Purpan, CHU de Toulouse, Toulouse, France. ${ }^{21}$ Centre de Référence du Syndrome de Marfan et des syndromes apparentés, Hôpital des Enfants, CHU de Toulouse, Toulouse, France. ${ }^{22}$ Plateforme de Transfert en Biologie Cancérologique, Centre Georges François Leclerc - UNICANCER- Institut GIMI, Dijon, France.

Received: 31 August 2021 Accepted: 14 November 2021 Published online: 04 December 2021

\section{References}

1. Parrini E, Ramazzotti A, Dobyns WB, Mei D, Moro F, Veggiotti P, et al. Periventricular heterotopia: phenotypic heterogeneity and correlation with Filamin A mutations. Brain J Neurol. 2006;129(Pt 7):1892-906.

2. Lange M, Kasper B, Bohring A, Rutsch F, Kluger G, Hoffjan S, et al. 47 patients with FLNA associated periventricular nodular heterotopia. Orphanet J Rare Dis. 2015;10:134.

3. Guerrini R, Mei D, Sisodiya S, Sicca F, Harding B, Takahashi Y, et al. Germline and mosaic mutations of FLN1 in men with periventricular heterotopia. Neurology. 2004;63(1):51-6.

4. Cannaerts E, Shukla A, Hasanhodzic M, Alaerts M, Schepers D, Van Laer L, et al. FLNA mutations in surviving males presenting with connective tissue findings: two new case reports and review of the literature. BMC Med Genet. 2018;19(1):140.

5. Ritelli M, Morlino S, Giacopuzzi E, Carini G, Cinquina V, Chiarelli N, et al. Ehlers-Danlos syndrome with lethal cardiac valvular dystrophy in males carrying a novel splice mutation in FLNA. Am J Med Genet A. 2017;173(1):169-76

6. Hommel AL, Jewett T, Mortenson M, Caress JB. Juvenile muscular atrophy of the distal upper extremities associated with $\mathrm{x}$-linked periventricular heterotopia with features of Ehlers-Danlos syndrome. Muscle Nerve. 2016;54(4):794-7.

7. Iwamoto DV, Huehn A, Simon B, Huet-Calderwood C, Baldassarre M, Sindelar CV, et al. Structural basis of the Filamin A actin-binding domain interaction with F-actin. Nat Struct Mol Biol. 2018;25(10):918-27.

8. Nakamura F, Osborn TM, Hartemink CA, Hartwig JH, Stossel TP. Structural basis of Filamin A functions. J Cell Biol. 2007;179(5):1011-25. 
9. Sheen VL, Jansen A, Chen MH, Parrini E, Morgan T, Ravenscroft R, et al. Filamin A mutations cause periventricular heterotopia with Ehlers-Danlos syndrome. Neurology. 2005;64(2):254-62.

10. Gómez-Garre P, Seijo M, Gutiérrez-Delicado E, del Castro Río M, de la Torre C, Gómez-Abad C, et al. Ehlers-Danlos syndrome and periventricular nodular heterotopia in a Spanish family with a single FLNA mutation. J Med Genet. 2006;43(3):232-7.

11. Sheen VL, Walsh CA. Periventricular heterotopia: new insights into EhlersDanlos syndrome. Clin Med Res. 2005;3(4):229-33.

12. Malfait F, Francomano C, Byers P, Belmont J, Berglund B, Black J, et al. The 2017 international classification of the Ehlers-Danlos syndromes. Am J Med Genet C Semin Med Genet. 2017;175(1):8-26.

13. Richards S, Aziz N, Bale S, Bick D, Das S, Gastier-Foster J, et al. Standards and guidelines for the interpretation of sequence variants: a joint consensus recommendation of the American College of Medical Genetics and Genomics and the Association for Molecular Pathology. Genet Med Off J Am Coll Med Genet. 2015;17(5):405-24.

14. Chen MH, Choudhury S, Hirata M, Khalsa S, Chang B, Walsh CA. Thoracic aortic aneurysm in patients with loss of function Filamin A mutations: clinical characterization, genetics, and recommendations. Am J Med Genet A. 2018;176(2):337-50.

15. de Wit MCY, de Coo IFM, Lequin MH, Halley DJJ, Roos-Hesselink JW, Mancini GMS. Combined cardiological and neurological abnormalities due to Filamin A gene mutation. Clin Res Cardiol Off J Ger Card Soc. 2011;100(1):45-50.

16. Hoashi T, Shiraishi I, Ichikawa H. Surgical experience for prolapse of both atrioventricular valves in a patient with Filamin A mutation. Cardiol Young. 2015;25(2):365-7.

17. Masurel-Paulet A, Haan E, Thompson EM, Goizet C, Thauvin-Robinet C, Tai A, et al. Lung disease associated with periventricular nodular heterotopia and an FLNA mutation. Eur J Med Genet. 2011:54(1):25-8.

18. Kinane TB, Lin AE, Lahoud-Rahme M, Westra SJ, Mark EJ. Case 4-2017. A 2-month-old girl with growth retardation and respiratory failure. N Engl J Med. 2017;376(6):562-74.

19. Eltahir S, Ahmad KS, Al-Balawi MM, Bukhamsien H, Al-Mobaireek K, Alotaibi W, et al. Lung disease associated with Filamin A gene mutation: a case report. J Med Case Rep. 2016;10:97.

20. Sasaki E, Byrne AT, Phelan E, Cox DW, Reardon W. A review of Filamin A mutations and associated interstitial lung disease. Eur J Pediatr. 2019;178(2):121-9.

21. Burrage LC, Guillerman RP, Das S, Singh S, Schady DA, Morris SA, et al. Lung transplantation for FLNA-associated progressive lung disease. J Pediatr. 2017;186:118-123.e6.

22. Fergelot P, Coupry I, Rooryck C, Deforges J, Maurat E, Solé G, et al. Atypical male and female presentations of FLNA-related periventricular nodular heterotopia. Eur J Med Genet. 2012;55(5):313-8.

23. Oda H, Sato T, Kunishima S, Nakagawa K, Izawa K, Hiejima E, et al. Exon skipping causes atypical phenotypes associated with a loss-of-function mutation in FLNA by restoring its protein function. Eur J Hum Genet EJHG. 2016;24(3):408-14.

24. Reinstein E, Frentz S, Morgan T, García-Miñaúr S, Leventer RJ, McGillivray $G$, et al. Vascular and connective tissue anomalies associated with X-linked periventricular heterotopia due to mutations in Filamin A. Eur J Hum Genet EJHG. 2013;21(5):494-502.

25. Solé G, Coupry I, Rooryck C, Guérineau E, Martins F, Devés S, et al. Bilateral periventricular nodular heterotopia in France: frequency of mutations in FLNA, phenotypic heterogeneity and spectrum of mutations. J Neurol Neurosurg Psychiatry. 2009;80(12):1394-8.

26. de Wit MCY, Kros JM, Halley DJJ, de Coo IFM, Verdijk R, Jacobs BC, et al. Filamin A mutation, a common cause for periventricular heterotopia, aneurysms and cardiac defects. J Neurol Neurosurg Psychiatry. 2009;80(4):426-8.

27. leda D, Hori I, Nakamura Y, Ohshita H, Negishi Y, Shinohara T, et al. A novel truncating mutation in FLNA causes periventricular nodular heterotopia, Ehlers-Danlos-like collagenopathy and macrothrombocytopenia. Brain Dev. 2018;40(6):489-92.

28. LaPointe MM, Spriggs EL, Mhanni AA. Germline mosaicism in X-linked periventricular nodular heterotopia. BMC Neurol. 2014;14:125.

29. de Wit MCY, Tiddens HAWM, de Coo IFM, Mancini GMS. Lung disease in FLNA mutation: confirmatory report. Eur J Med Genet. 2011;54(3):299-300.
30. Jenkins ZA, Macharg A, Chang C-Y, van Kogelenberg M, Morgan T, Frentz $S$, et al. Differential regulation of two FLNA transcripts explains some of the phenotypic heterogeneity in the loss-of-function filaminopathies. Hum Mutat. 2018;39(1):103-13.

31. Clapham KR, Yu TW, Ganesh VS, Barry B, Chan Y, Mei D, et al. FLNA genomic rearrangements cause periventricular nodular heterotopia. Neurology. 2012;78(4):269-78.

32. Jefferies JL, Taylor MD, Rossano J, Belmont JW, Craigen WJ. Novel cardiac findings in periventricular nodular heterotopia. Am J Med Genet A. 2010;152A(1):165-8.

33. Nurden P, Debili N, Coupry I, Bryckaert M, Youlyouz-Marfak I, Solé G, et al. Thrombocytopenia resulting from mutations in Filamin A can be expressed as an isolated syndrome. Blood. 2011;118(22):5928-37.

34. Walsh M, Hebbard G, Trainer A. Update on a previously reported male with a FLNA missense mutation. Eur J Hum Genet. 2017;25(8):905-6.

35. Parrini E, Mei D, Wright M, Dorn T, Guerrini R. Mosaic mutations of the FLN1 gene cause a mild phenotype in patients with periventricular heterotopia. Neurogenetics. 2004;5(3):191-6.

36. Sheen VL, Dixon PH, Fox JW, Hong SE, Kinton L, Sisodiya SM, et al. Mutations in the $X$-linked filamin 1 gene cause periventricular nodular heterotopia in males as well as in females. Hum Mol Genet. 2001;10(17):1775-83.

37. Shah AS, Black ED, Simon DM, Gambello MJ, Garber KB, lannucci GJ, et al. Heterogeneous pulmonary phenotypes in Filamin A mutation-related lung disease. Pediatr Allergy Immunol Pulmonol. 2021;34(1):7-14.

38. Campens L, Demulier L, De Groote K, Vandekerckhove K, De Wolf D, Roman MJ, et al. Reference values for echocardiographic assessment of the diameter of the aortic root and ascending aorta spanning all age categories. Am J Cardiol. 2014;114(6):914-20.

\section{Publisher's Note}

Springer Nature remains neutral with regard to jurisdictional claims in published maps and institutional affiliations.

Ready to submit your research? Choose BMC and benefit from

- fast, convenient online submission

- thorough peer review by experienced researchers in your field

- rapid publication on acceptance

- support for research data, including large and complex data types

- gold Open Access which fosters wider collaboration and increased citations

- maximum visibility for your research: over $100 \mathrm{M}$ website views per year

At BMC, research is always in progress.

Learn more biomedcentral.com/submissions 